\title{
The simulation study of three typical time frequency analysis methods
}

\author{
Yifeng $\mathrm{Li}^{\mathrm{a}}$, Lihui Zhang, Baohui Li, Yan Xu, Sanyuan Wu, Xiaoyang Wei, Xiaoyan Liu, Rong Lin and \\ Quan Wang \\ Institute of Aviation Medicine, Air Force, 100142, Beijing, China
}

\begin{abstract}
The principals and characteristics of three typical time frequency analysis methods that Short Time Furious transformation, wavelet transformation and Hilbert-Huang transformation are introduced, and the mathematical definition, characteristics and application ranges of these analysis methods and so on are pointed out, then their time-frequency local performance is made analysis and comparison through computer programming and simulation.
\end{abstract}

\section{Introduction}

In the field of Aeronautics and Astronautics, in order to prevent pilots from air syncope in flying and to get a better flight, the human physiological signals under $+\mathrm{Gz}$ acceleration need to be monitored and studied. The recognition, feature extraction and monitoring analysis and other technologies of variety of physiological signals including EEG signal has become a hot topic of research in recent years [1]. All kinds of analysis methods such as Fourier transformation [2-4], Short Time Fourier transformation[5-7],wavelet and wavelet package transformation[8-12], Hilbert-Huang Transformation (HHT) [13-17] and other technologies have been applied to analysis of signal. Fourier transformation is the basic of analysis of time domain and frequency domain for signal, and is very valid to the analysis of periodic stable signal. But in nature and engineering areas there are a large number of non periodic non stationary signal, and EEG is typical non periodic signal, so the classic analysis method based on Fourier transformation can not accurately reflect the local time-varying frequency spectral characteristic of signal, unable to obtain many of the key information. So a series of new signal time frequency analysis methods are put forward and developed. The basic idea of time frequency analysis is to design the joint function of time and frequency, and at the same time, the density and intensity of energy of signal at different time and frequency is described. Before the analysis and comparison on time-frequency performance of various of methods has been seen, but the research on the comparison and analysis on Short Time Fourier transformation, wavelet transformation, and HHT, this three kinds of methods is rare through the designed simulation signal. This study first briefly introduces of the principles and characteristics of this several methods, Short Time Fourier transformation, wavelet transformation and HHT, then EEG signal is simulated by the designed suitable simulation signal through programming, so the time frequency graphs are made out through simulation signal, and time frequency performance of three methods is made a contrast and analysis, to explore the application prospect of them in processing and analysis of dynamic EEG signal.

\footnotetext{
${ }^{a}$ Corresponding author: liyf8886@sina.com
} 


\section{The introduction of basic principle and method}

\subsection{Short Time Furious transformation (STFT)}

STFT assumes non stationary signal as that every small segment is smooth, intercepts signal through using a sliding window, and makes Fourier transformation for the intercepted signal again and again, so as to obtain the frequency spectrum of signal at any time. Its definition is as:

$$
\operatorname{STFT}(t, f)=\int x(t) h(-t) e^{-j \pi f} d t
$$

The advantage of STFT is that its physical meaning is clear and there is no cross term appearing. It is a time frequency analysis method which application is the most. According to the Heisenberg uncertainty principle, the product of time width and bandwidth of signal should be a constant relevant to sampling rate. In other words, when time resolution is needed to improve, the resolution of frequency is often needed to sacrifice, and vice versa. When the analyzed signal contains many kinds of types of scale components with large differences, STFT is powerless. Although STFT there is the defects such as resolution ratio is not high and so on, its algorithm is simple, and easy to implement, so in a long time it is a powerful tool of non stationary signal analysis is, and still is widely used.

\subsection{Wavelet transformation}

Wavelet transform, as a kind of new linear time frequency analysis method, is developed in the Middle and late period of the 1980s of 20th century. It makes multi-scales analysis of signal by the operation function of dilation and translation and others. It is a milestone development in reconcile analysis development history. The wavelet transformation definition of $x(t)$ is as:

$$
\begin{aligned}
& W T_{x}(\alpha, \tau)=\frac{1}{\sqrt{a}} \int x(t) \phi^{*}\left(\frac{t-\tau}{\alpha}\right) d t \\
& =\left\langle x(t), \phi_{\alpha \tau}(t)\right\rangle
\end{aligned}
$$

It is the inner product result of function and wavelet basis , and among this wavelet basis:

$$
\varphi_{\alpha \tau}=\frac{1}{\sqrt{\alpha}} \varphi\left(\frac{t-\tau}{\alpha}\right)
$$

$\alpha$ is the scale parameter, and $\tau$ is the translation parameter. The wavelet basis is different with the difference of $\alpha$ and $\tau$, and it can describe the frequency band characteristic of signal, that is it can show time-frequency characteristic of signal at the same time, and can very sensitively detect mutation of signal. Wavelet transformation is also subjected to the Heisenberg uncertainty principle, but the window of wavelet transformation is adjustable time-frequency window, using a narrow window in high frequency, and using a wide window in low frequency, fully embodying the idea of multi-resolutions analysis, and this is consistent with the feature of time varying non stationary signal is. The characteristic of multi scales and multi resolutions makes wavelet transformation be known as "mathematical microscope". But wavelet transformation does not possess characteristic of adaptive decomposition, and the finite length of wavelet basic can also cause energy leakage, and its selection principle is still a difficult problem. After development of about 30 years, wavelet transform has made breakthrough progress, and formed the multi resolutions analysis, framework and filter group-this three big complete and abundant wavelet transformation theory system, and now it has been widely used in signal elimination noise, medicine and many other areas. 


\subsection{Hilbert-Huang transformation (HHT)}

Hilbert Huang transformation is a new signal processing method proposed by N.E.Huang et al. in 1998, and it is considered as a major breakthrough of linear and steady-state spectral analysis based on Furious transformation in recent years. Essentially it is the process that making smoothing processing for signal, and decomposing stepwise the wave of different scales in signal, formatting intrinsic mode function component, then making Hilbert Huang transformation for these components. HHT method consists of two main steps:

(1)The original data is made preprocess, and through empirical mode decomposition (EMD) method, the data is decomposed into intrinsic mode function (IMF) of $\mathrm{n}$ order, that is:

$$
x(t)=\sum_{i=1}^{n} c_{i}+r_{n}
$$

(2)The decomposed each order IMF component is made Hilbert Huang transformation, and the respective instantaneous frequency is got, then the time frequency diagram is made.

Hilbert Huang transformation is an effective tool for nonlinear and non stationary data analysis, and it is a self-adaptive treatment method. It's the biggest characteristic is that by EMD decomposition of signal, making non stationary signal smooth, resulting in instantaneous frequency has physical meaning, and then exporting the meaningful Hilbert time frequency spectrum. The analysis basic of HHT is: the data structure of the modulation mechanism of intra wave and no fixed basement (that is IMF characteristic). It doesn't take that time and frequency is absolutely two space variables, and no longer believes that the fundamental component part of signal is complete sine function, namely in the basic component part, instantaneous frequency is also changing with time, but it is worth noting, now the proof of HHT obviously falls after its application. The further development of HHT also requires that the envelope problem, EMD decomposition theory verifying, the end point effect suppression in boundary treatment and other issues have a better way to solve. Its theoretical perfection will be a long process. While this does not affect its application. In just a few years from the method of Hilbert Huang transformation publishes up to now, HHT method has always subjected an extensive attention of scholars at home and abroad and it has been widely used in biomedicine, equipment diagnosis and other various of scientific research and engineering field.

\section{Simulation signal contrast and analysis study}

In order to better contrast the treatment effects of Short Time Fourier transformation, wavelet transformation and Hilbert Huang transformation, this several methods on EEG data, and taking into account the basic rhythms signals in EEG [18], that is EEG is composed of low frequency and high frequency signals, so the segmented two sine functions are used to simulate the dynamic EEG data, frequency is respectively taken as $10 \mathrm{~Hz}$ and $50 \mathrm{~Hz}$, and sampling frequency is taken as $1000 \mathrm{~Hz}$, taking data of $2 \mathrm{~s}$. The time point of mutation is taken at the time of about $1 \mathrm{~s}$. The simulation signal is as:

$$
x(t)=\left\{\begin{array}{cc}
\sin (20 \pi t) & 0 \leq t \leq 1 s \\
\sin (100 \pi t) & 1<t \leq 2 s
\end{array}\right.
$$

STFT, wavelet transformation, and HHT, this several kinds of transformation treatment are respectively made for this simulation signal, and the time frequency graphs of this several kinds of transformation are made out. The simulation graph and time frequency graphs of this several kinds of transformation are shown in the following graphs. 


\section{ICMSB2016}

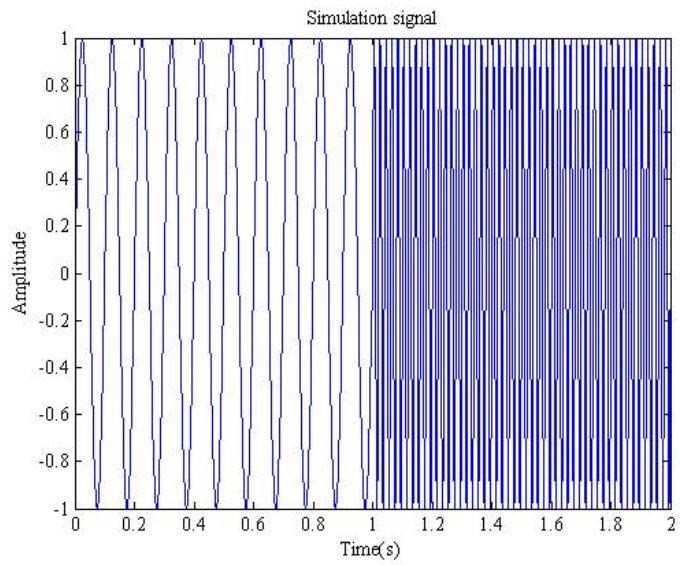

Figure 1. The simulation signal.

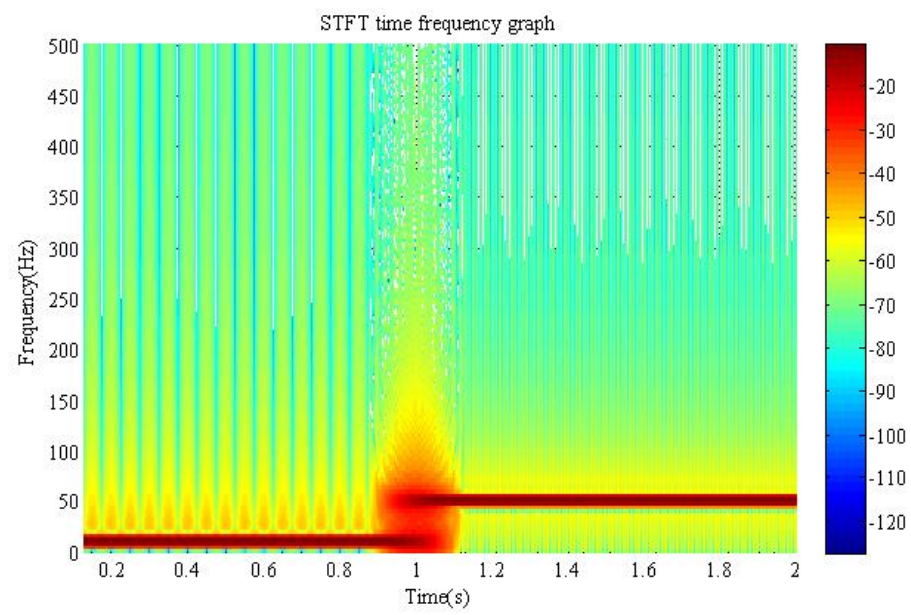

Figure 2. STFT time frequency graph.

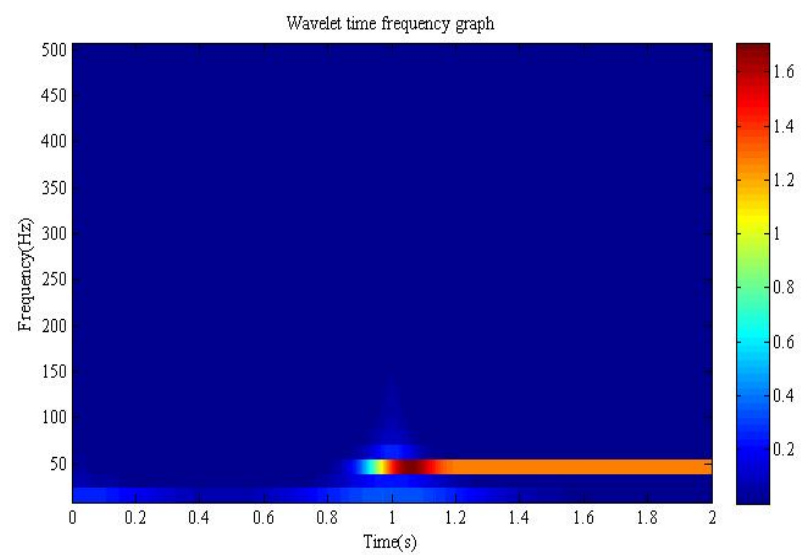

Figure 3. Wavelet time frequency graph. 


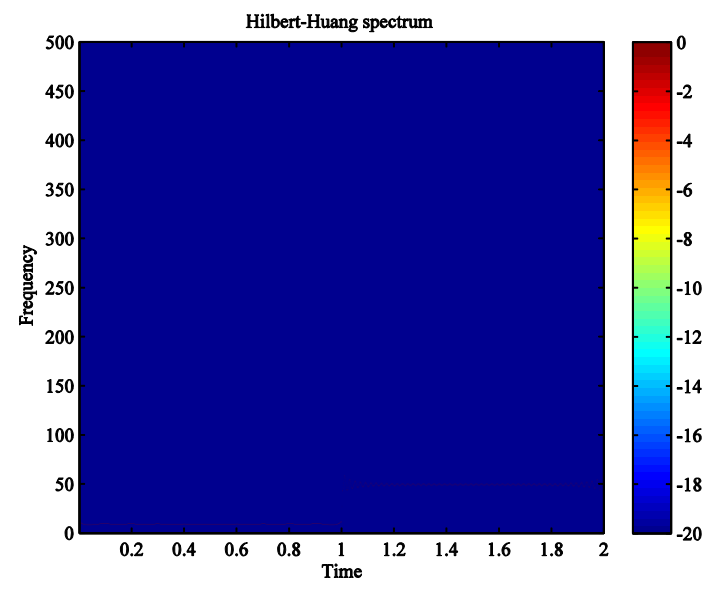

Figure 4. HHT spectrum graph.

Three methods all can distinguish the change of main frequency. From Figure 2, spectrum graph of Short Time Furious transformation it can be seen that, although this figure can distinguish the moment of two main frequency components and mutation occurrence, the resolution is not high. According to the Heisenberg uncertainty principle, the product of time width and bandwidth of signal should be a constant relevant to sampling rate. In other words, when time resolution is needed to improve, the resolution of frequency is often needed to sacrifice, and vice versa. For EEG signal, when the frequency resolution is required, the time window length is likely to appear too large, and at this time FFT may be difficult to meet the requirement. The treatment effect is not satisfactory for non-stationary nonlinear signal such as EEG. Figure 3 is a wavelet spectrum. Although the timefrequency resolution of wavelet transformation are both very good, for the smaller frequency component, the time point of frequency hopping is described not enough accurately. Figure 4 is the HHT spectrum. It can be seen from the figure that processing effect of HHT on nonlinear unstable signal has a unique advantage, because this method does not exist the steps of partition and translation of Windows when using, adopting that separately making analysis for the short time series. Therefore, it makes a good balance between the requirements of time resolution and frequency resolution, that is to say, as long as it meets the Shannon theorem (i.e., sampling law), this method can improve time domain resolution as much as possible under the situation that without sacrificing frequency domain resolution. HHT spectrum displays the distribution of signal energy in time frequency domain, and can help more clearly understand the distribution situation of low and high frequency part in signal. Moreover, HHT method structures basis function from the signal itself, directly reflects the characteristic of signal itself, and eliminate the generated virtual volume due to the mismatch of base function and signal in transformation process. so the obtained HHT spectrum can not only locate the position of frequency mutation point, but also can accurately distinguish two main frequency components of $10 \mathrm{~Hz}$ and $50 \mathrm{~Hz}$, and contains no crossing interference term, with more superior ability of detecting signal singularity than FFT and wavelet transformation .In addition, HHT can use some unique forms, such as instantaneous energy spectrum, time frequency spectrum and others to display new characteristic. But relatively speaking, this method quite consumes computer memory and calculation time, that is, relatively calculation is slower, and this has limitation in practical application. Moreover, envelope rationality, EMD decomposition theory verifying, and end point effect restrain in boundary treatment and other issues still need further to be proofed, namely relatively speaking the theoretical development of this method is not as perfect as other two, and its theory perfection will be a longer process relatively. 


\section{Conclusions}

This study introduces the principal and method of STFT, wavelet transformation, and HHT, three typical time-frequency analysis methods, and the time frequency local performances of this several methods are made analyzed and compared through computer simulation, Then the advantages and disadvantages of this several methods are discussed. EEG is a typical time varying, non stationary signal, time-frequency union analysis technology is created and developed according to the need of real science application. Three kinds of methods all can better distinguish the main frequency, but relatively speaking, HHT method, has a good time-frequency and adaptability for signal. But this method also has its limitations. It is believed that with the continuous perfection and development of modern time frequency analysis theory and technology, in the field of EEG monitoring and analysis of aeromedicine, time frequency union analysis technology will have a more broad application prospect and greater development potential.

\section{References}

1. Koji Morikawa, Akinori Matsumoto, Shrishail Patki, Bernard Grundlehner,Auryn Verwegen, Jiawei Xu, Srinjoy Mitra and Julien Penders, Compact Wireless EEG Sys tem with Active Electrodes for Daily Healthcare Monitoring, IEEE International Conference on Consumer Electronics (ICCE).2013:204-205.

2. SN. Tang, FC. Jan, HW. Cheng, CK. Lin, G Z. Wu, Multimode Memory-Based FFT Processor for Wireless Display FD-OCT Medical Systems, IEEE Transactions on Circuits and Systems I: Regular Papers.2014; 61 ( 12): 3394 - 3406.

3. Jing Han, Lingling Zhang, G. Leus, Partial FFT Demodulation for MIMO-OFDM Over TimeVarying Underwater Acoustic Channels, IEEE Signal Processing Letters.2016: 23 (2 ): 282 - 286.

4. T. Yang,H. Pen , Z. Wang, C. S. Chang, Feature Knowledge Based Fault Detection of Induction Motors Through the Analysis of Stator Current Data, IEEE Transactions on Instrumentation and Measurement.2016;65 ( 3 ): 549 - 558.

5. W. Liu, X. Zhang, Signal modulation characteristic analysis based on SFFT-Haar algorithm, IET International Radar Conference 2013, Xi'an, 2013: 1-4.

6. B. Kim, S.H. Kong, S. Kim, Low Computational Enhancement of STFT-Based Parameter Estimation, IEEE Journal of Selected Topics in Signal Processing, 2015, 9(8 ):1610 - 1619 .

7. K. Jaganathan, Y. C. Eldar, B. Hassibi, STFT Phase Retrieval: Uniqueness Guarantees and Recovery Algorithms, IEEE Journal of Selected Topics in Signal Processing, 2016,10 (4) :770 781 .

8. R. Mothi, M. Karthikeyan, A wavelet packet and fuzzy based digital image watermarking, 2013 IEEE International Conference on Computational Intelligence and Computing Research (ICCIC), Enathi, 2013:1 - 5.

9. J.S Pan, and L. Yue, "A ridge extraction algorithm based on partial differential equations of the wavelet transform, "2014 IEEE Symposium on Computational Intelligence for Multimedia, Signal and Vision Processing (CIMSIVP), Orlando, FL, pp.1 - 5, 2014.

10. S.A.R. Naqvi, I. Touqir, and S.A. Raza, "Adaptive geometric wavelet transform based two dimensional data compression, "2013 7th IEEE GCC Conference and Exhibition (GCC), Doha , pp. $583-588,2013$.

11. G. Serbes, N. Aydin, and H.O. Gulcur, "Directional dual-tree complex wavelet packet transform, "2013 35th Annual International Conference of the IEEE, Engineering in Medicine and Biology Society (EMBC), Osaka, pp.3046-3049, 2013.

12. S. Bharkad, and M. Kokare, "Fingerprint matching using discreet wavelet packet transform , "2013 IEEE 3rd International Advance Computing Conference (IACC), Ghaziabad, pp.1183-1188, 2013. 
13. K. Rai , V. Bajaj ,A. Kumar, Hilbert-Huang transform based classification of sleep and wake EEG signals using fuzzy c-means algorithm, 2015 International Conference on Communications and Signal Processing (ICCSP), IEEE, Melmaruvathur, 2015: 0460 - 0464.

14. Y. Wang,C. Chen, H. He, Large Eddy Simulation and Hilbert Huang Transform for fluctuation pressure of high speed train, 2015 IEEE International Instrumentation and Measurement Technology Conference (I2MTC), Pisa, 2015: $284-288$.

15. Rahul Kumar Chaurasiya, K. Jain, S. Goutam ,Manisha, Epileptic seizure detection using HHT and SVM, 2015 International Conference on Electrical, Electronics, Signals, Communication and Optimization (EESCO), Visakhapatnam, 2015: $1-6$.

16. K. Y. Lin, D. Y. Chen, W. J. Tsai, Face-Based Heart Rate Signal Decomposition and Evaluation Using Multiple Linear Regression, IEEE Sensors Journal ,2016; 16 ( 5 ): 1351 - 1360.

17. P. Y. Chen ,Y. C. Lai , J. Y. Zheng, Hardware Design and Implementation for Empirical Mode Decomposition, IEEE Transactions on Industrial Electronics,2016; PP ( 99 ): 1.

18. M. Nakamura, Q. Chen, T. Sugi, A. Ikeda, and H. Shibasaki, "Technical quality evaluation of EEG recording based on electroencephalographers' knowledge,"Medical Engineering \& Physics, vol. (27),2005, pp.93-100. 